The Glasgow Sensory Questionnaire: validation of a French language version and refinement of sensory profiles of people with high Autism-Spectrum Quotient

Laurie-Anne Sapey-Triomphe ${ }^{1,2}$, Annie Moulin ${ }^{1,2}$, Sandrine Sonié ${ }^{1,2,3,4}$, Christina Schmitz ${ }^{1,2}$

1. Lyon Neuroscience Research Centre, Brain Dynamics and Cognition Team, CNRS UMR5292, INSERM U1028; University Lyon 1, Lyon, France.

2. Université de Lyon, Lyon, France.

3. Centre de Ressource Autisme Rhône-Alpes, Centre Hospitalier Le Vinatier, Bron, France.

4. Hôpital Saint-Jean-de-Dieu, Lyon, France. 


\section{Abstract}

Sensory sensitivity peculiarities represent an important characteristic of Autism Spectrum Disorders (ASD). We first validated a French language version of the Glasgow Sensory Questionnaire (GSQ) (Robertson and Simmons 2013). The GSQ score was strongly positively correlated with the Autism-Spectrum Quotient (AQ) $\left(r=.81, p<10^{-6}, n=245\right)$. We further examined sensory profiles of groups with high versus low AQ. The high AQ group scored higher at the GSQ than the low AQ group for every sensory modality. Moreover, the high AQ group showed greater consistency in their patterns of hypersensitivity and hyposensitivity between sensory modalities, and stronger correlations between hyper and hyposensitivity. Results are discussed in the context of theories accounting for atypical sensory perception in ASD.

Keywords : Autism spectrum disorder, Autism Quotient, Glasgow Sensory Questionnaire, hypersensitivity, cross-cultural adaptation.

\section{Corresponding author:}

Correspondence concerning this article should be addressed to Dr Christina Schmitz

Email : christina.schmitz@inserm.fr

Phone: 0472138911 


\title{
The Glasgow Sensory Questionnaire: validation of a French language version and refinement of sensory profiles of people with
}

\section{high Autism-Spectrum Quotient}

\begin{abstract}
Sensory sensitivity peculiarities represent an important characteristic of Autism Spectrum Disorders (ASD). We first validated a French language version of the Glasgow Sensory Questionnaire (GSQ) (Robertson and Simmons 2013). The GSQ score was strongly positively correlated with the Autism-Spectrum Quotient (AQ) $\left(r=.81, p<10^{-6}, n=245\right)$. We further examined sensory profiles of groups with high versus low AQ. The high AQ group scored higher at the GSQ than the low AQ group for every sensory modality. Moreover, the high AQ group showed greater consistency in their patterns of hypersensitivity and hyposensitivity between sensory modalities, and stronger correlations between hyper and hyposensitivity. Results are discussed in the context of theories accounting for atypical sensory perception in ASD.
\end{abstract}

Keywords : Autism Spectrum Disorder, Autism-Spectrum Quotient, Glasgow Sensory Questionnaire, hypersensitivity, cross-cultural adaptation. 


\section{Introduction}

Autism Spectrum Disorder (ASD) is primarily characterized by deficits in social communication and interactions, and by restricted and repetitive patterns of behaviors, interests or activities. Yet, sensory abnormalities were added as a core symptom of ASD in the last version of the Diagnostic and Statistical Manual of Mental Disorders (DSM-5, American Psychiatric Association 2013). Indeed, a new criterion emerged in the restrictive and repetitive behavior section: one of the features is now having hyper- or hypo-reactivity to sensory inputs or unusual interest in sensory aspects of the environment. Interestingly, sensory abnormalities had been already noted by Kanner and Asperger in their early descriptions of autism (Asperger 1944; Frith 1991; Kanner 1943). As ASD diagnosis is now clearly associated with atypical sensory sensitivity, it seems important to better describe and quantify peculiarities in each sensory domain.

Hyposensitivity and hypersensitivity are both present in ASD (Baranek et al. 2006; Baum et al. 2015), and are associated with under- or over-responsiveness to sensory stimuli (Green et al. 2013; Haigh et al. 2016; Tavassoli et al. 2014), as well as sensation seeking or avoidance (Ashburner et al. 2013; Baranek et al. 2006; Crane et al. 2009). Additionally, unusual sensory interests were associated with more severe autistic symptoms (Zachor and Ben-Itzchak 2013), as measured with the ADI (Le Couteur et al. 2003) and ADOS (Lord et al. 2000). Atypical sensory sensitivity has been described in every sensory modality (Baum et al. 2015; Foxe et al. 2015; Kern et al. 2007; Marco et al. 2011): in the visual (Simmons et al. 2009), auditory (Haesen et al. 2011; Sinclair et al. 2016), olfactory (Bennetto et al. 2007; Dudova et al. 2011; Wicker et al. 2016), gustatory (Bennetto et al. 2007; Tavassoli and BaronCohen 2012), tactile (Blakemore et al. 2006; Cascio et al. 2008; Güçlü et al. 2007; Puts et al. 2014), vestibular (Kern et al. 2007) and proprioceptive (Riquelme et al. 2016) modalities. 
Hyper-reactivity to sensory inputs has been one of the main findings. More than $90 \%$ of the individuals with ASD would be concerned with sensory abnormalities (Crane et al. 2009; Leekam et al. 2007; Marco et al. 2011).

Theories accounting for an abnormal sensory sensitivity in ASD mostly focus on sensory hypersensitivity, and often fail to explain both hypersensitivity and hyposensitivity. The Enhanced Perceptual Functioning (EPF) theory of ASD suggested increased perceptual ability in ASD (Mottron et al. 2006; Mottron and Burack 2001). In this view, sensory hypersensitivity could contribute to superior discrimination performance or peaks of ability found in ASD. Besides, the Intense World Syndrome theory suggested that sensory hypersensitivity could be related to excessive neural processing and neural hyper-reactivity (Markram et al. 2007; Markram and Markram 2010). Furthermore, theories suggesting either an increased or decreased level of endogenous neural noise in ASD could lead to hypo- or hyper-sensitivity (Dakin and Frith 2005; Davis and Plaisted-Grant 2015; Simmons and Milne 2015; Simmons et al. 2007). Bayesian theories propose that peculiar sensory sensitivity in ASD could be due to an atypical relative weighting of the confidence in incoming sensations and the confidence associated with sensory predictions, in a given context (Brock 2012; Haker et al. 2016; Lawson et al. 2014; Palmer et al. 2017; Pellicano and Burr 2012; Van de Cruys et al. 2013, 2014, 2016). Within this framework, the predictability of the sensation plays a central role. The presence of both hypersensitivity and hyposensitivity in ASD (or in people with high autistic traits) would suggest that the atypical reactivity to sensory stimulations in ASD would be contextual, rather than uniformly characterized by a higher/lower sensitivity of the receptors and/or perceptual systems.

Relationships between sensory sensitivity peculiarities and autistic traits have been often investigated using self-administered sensory questionnaires and the Autism-Spectrum Quotient (Horder et al. 2014; Robertson and Simmons 2013; Takayama et al. 2014; Tavassoli 
et al. 2014; Ward et al. 2017). The Autism-Spectrum Quotient (AQ) (Baron-Cohen et al. 2001) is a self-administered questionnaire, which is probably the most widely used questionnaire assessing autistic traits in adults with no intellectual disability. The AQ is made of 50 items assessing social skills, attention switching, attention to details, communication and imagination. As the AQ has adequate levels of specificity and sensitivity, it allows targeting individuals with high AQ who would also meet the criteria for ASD diagnosis in large sample data (Baron-Cohen et al. 2001; Lundqvist and Lindner 2017; Sonié et al. 2013). Limitations of the AQ include the relatively low internal consistency of its subscores, as well as results showing that the AQ does not meet all criteria for a unidimensional scale of autistic traits (Lundqvist and Lindner 2017).

The AQ is strongly positively correlated with the Glasgow Sensory Questionnaire (GSQ - a sensory sensitivity questionnaire) in participants with low and high AQ (Robertson and Simmons 2013), or in neurotypical and ASD individuals (Takayama et al. 2014; Ward et al. 2017). Particularly, Horder and colleagues (Horder et al. 2014) showed that the AQ was more strongly correlated with the GSQ (Robertson and Simmons 2013) than with the Adult/Adolescent Sensory Profile (AASP) (Brown and Dunn 2002). The GSQ is a 42-item self-questionnaire measuring the frequency of atypical sensory processing events (Robertson and Simmons 2013). It investigates both hypersensitivity and hyposensitivity in the visual, auditory, olfactory, gustatory, tactile, vestibular and proprioceptive modalities. Contrary to the AASP which was not specifically developed for individuals with ASD, the GSQ targets sensory experiences that can be encountered in the daily-life of people with ASD or high AQ. In addition, the GSQ assesses seven sensory modalities, whereas the AASP investigates only five sensory modalities. Other existing tools assessing sensory sensitivity include the recent Sensory Perception Quotient (Tavassoli et al. 2014), but which does not allow the investigation of the seven sensory modalities and which does not weight equally each sensory 
modality. The Cardiff Anomalous Perceptions Scale (CAPS) has also been related to autistic traits (Horder et al. 2014; Milne et al. 2017), but it targets perceptual abnormalities in a context of psychosis and includes items assessing sensory sensitivity but also many unrelated items such as those dealing with hallucinations. In the present study, as we aimed at measuring sensory hypersensitivity and hyposensitivity in each of the seven sensory modalities, we prioritized the use of the GSQ.

Previous studies on the GSQ did not detail its internal structure, nor the scores of hypersensitivity and hyposensitivity separately, per sensory modality (Robertson and Simmons 2013; Takayama et al. 2014). This aspect would be useful to better characterize sensory profiles in groups of individuals with low and high AQ. Refining sensory profiles in ASD could help determining whether hypersensitivity prevails in ASD, which could be consistent with the low endogenous noise theory of ASD (Davis and Plaisted-Grant 2015), the Intense World Syndrome theory (Markram et al. 2007; Markram and Markram 2010) or the EPF theory (Mottron et al. 2006; Mottron, and Burack 2001). It would also help showing whether sensory peculiarities increase linearly when the AQ increases, or whether atypical sensory profiles characterize people with high AQ or ASD. The second assumption would be associated with a distinct pattern of hyper and hyposensitivity GSQ scores across modalities in a high AQ group as compared to a low AQ group, and by a non-linear relationship between the AQ and GSQ scores across the whole AQ range. In particular, theories suggesting peaks of enhanced perceptual abilities in ASD (Mottron et al. 2006; Mottron, and Burack 2001) would be consistent with a more heterogeneous pattern of hypersensitivity in the high AQ group as compared to the low AQ group. Indeed, sensory expertise should be associated with heterogeneous profiles showing a heightened hypersensitivity score for a specific sensory modality. 
The structure of the GSQ and the fact that it was specifically developed to target the sensory issues associated with ASD make it a powerful tool to describe sensory profiles in ASD. It seems important to underline that sensory sensitivity, assessed by questionnaires like the GSQ, provide measurements of self-reported (subjective) reactivity to sensory stimulations, rather than objective measurements of sensitivity of the sensory systems (e.g. such as measures of perception threshold). As research on sensory sensitivity in ASD is a growing topic, it is important to have a tool that enables to individually quantify subjective sensitivity in behavioral or neuroimaging studies. In addition, it could help defining groups of participants with clearly established and/or homogeneous sensory profiles. Apart from research purposes, refining sensory sensitivity profiles could also contribute to define better sensory environments for individuals with high AQ or with ASD, by providing a somewhat comprehensive definition of their sensory sensitivity. As subjective reports show that unusual sensory experiences have a significant impact on the daily life of individuals with ASD (Kirby et al. 2014; Robertson and Simmons 2015), a tool quantifying these experiences is necessary.

The GSQ was originally designed in English (Robertson and Simmons 2013) and its Japanese translation has been validated (Takayama et al. 2014). The GSQ needs to be crossculturally adapted and translated to be used as a standard across international studies. The process of cross-cultural adaptation of a questionnaire involves more than a simple translation: according to the Universalist model of cross cultural adaptation of health questionnaires (Herdman et al. 1998), six types of equivalences between the original questionnaire and the translated questionnaire must be considered. Semantic equivalence relates to the translation and operational equivalence deals with the way the questionnaire can be presented, administered and scored. Item and conceptual equivalences deal with how the concepts are equivalent between one country and another, and this equivalence can be tested 
by comparing internal structures of the questionnaires obtained in several languages and/or countries. In order to validate the translation of a questionnaire, its internal structure should show the same main factors regardless of the country and/or language it is in. From the way the GSQ is constructed, we would expect either a structure reminding of the seven sensory modalities tested, or separating hyposensitivity from hypersensitivity items regardless of the sensory modality tested, or a mix of the two. The analysis of the internal structure would also potentially allow a distinction between populations, not only based on a global score, but also on differential relationships between the items of the questionnaire. Hence, we analyzed the GSQ internal structure on a high AQ group and of a low AQ group, using both factor analysis and hierarchical cluster analysis, the latter giving a unique picture of the dimensionality of a scale. As the GSQ was recently developed (2013), the GSQ internal structure has not been assessed yet, so we provide detailed factor analysis results to allow comparison with further cross-cultural adaptation studies of the GSQ. The last two equivalences, within the Universalist framework, comprise measurement equivalence, that deals with psychometric properties, indices of internal consistency and construct validity, and functional equivalence, that assesses the overall equivalence of results of questionnaires used in different languages/countries.

The present work had two main aims: (1) the validation of a French language version of the GSQ according to the Universalist model of cross-cultural adaptation (Herdman et al. 1998), and (2) the refinement of the sensory profiles of individuals with high or low AQ. To do so, both GSQ and AQ questionnaires were presented in the same population of adults. We specifically targeted neurotypical participants and participants suspected to have ASD. Aim 1 involved the exploration of the reliability, construct validity and internal structure of the questionnaire, and a comparison of results with the available studies using the GSQ (Horder et al. 2014; Robertson and Simmons 2013; Takayama et al. 2014; Ward et al. 2017). Aim 2 
involved the analysis of sensory sensitivity profiles in groups of participants with low or high AQ. The relationships between hypersensitivity and hyposensitivity scores were compared between groups in order to know whether the high AQ group would show a specific sensory sensitivity profile, besides from higher GSQ scores. Within groups, hyper- and hyposensitivity scores were compared in each of the seven sensory modalities to assess whether hyper or hyposensitivity predominated. Finally, the cluster and factor analyses performed in each group enabled a clearer description of the sensory profiles and of the GSQ response consistency in each group.

\section{Material and Methods}

\section{Participants}

This study involved 245 participants (114 females and 131 males, mean age: 32.1 \pm 10.8 years old). Demographic data are described in Table 1. Participants anonymously completed the AQ and the GSQ, as well as a short demographical questionnaire asking for age, education level (number of years of formal education), gender, and diagnosis (“ $A S D$ diagnosis", "no ASD diagnosis" and/or "other psychiatric or neurologic trouble"). Onehundred and forty-five participants reported having no diagnosis of ASD, and 95 participants reported having a formal ASD diagnosis. Inclusion criteria were being aged between 18 and 65 , declaring to not suffer from other psychiatric or neurological troubles, and having filled up the three questionnaires entirely. Participants were informed that it would take about 25 minutes to complete the three questionnaires. Questionnaires were first anonymously fulfilled by individuals with ASD who used to participate in our studies. One of the participants with ASD proposed to post a link to the questionnaires on her personal webpage, a blog on Asperger syndrome mostly followed by women with ASD. Finally, participants with no ASD 
diagnosis were participants who used to take part in our past studies, acquaintances of the laboratory members, and individuals who might have seen the questionnaires on the previously mentioned blog on Asperger syndrome (but who reported having no formal diagnosis of ASD).

\section{[Please, insert Table 1]}

\section{The Autism-Spectrum Quotient questionnaire}

The Autism-Spectrum Quotient (AQ) is a 50-item questionnaire assessing social skills, attention switching, attention to details, communication and imagination (Baron-Cohen et al. 2001). For items such as, "I find social situations easy", participants have to choose which answer best describes how the item applies to them: definitely agree, slightly agree, slightly disagree and definitely disagree. Depending on the items, the answers definitely agree and slightly agree score 1 point, and the answers slightly disagree and definitely disagree score 0 point, or vice-versa. Total scores can range from 0 to 50 . The higher the AQ is, the more autistic traits are found. In our study, participants completed the validated French version of the AQ (Sonié et al. 2011).

We used a standard AQ cut-off of 26 and above for the high AQ group, and an AQ below 26 for the low AQ group (Baron-Cohen et al. 2001; Sonié et al. 2013; Tavassoli et al. 2014). Sonié and colleagues (2013) showed that a cut-off score of 26 at the French version of the AQ distinguished ASD from control participants, with a sensitivity of $89 \%$ and a specificity of $98 \%$. In the present study, the low AQ group consisted of 143 participants, including 5 individuals who reported having a formal diagnosis of ASD. The high AQ group consisted of 102 participants, including 90 individuals who reported having a formal diagnosis of ASD. The demographical data of the two groups are described in Table 1. 


\section{The Glasgow Sensory Questionnaire (GSQ)}

The GSQ is a self-administered questionnaire for adults, investigating both hypersensitivity (21 questions) and hyposensitivity (21 questions) in seven sensory modalities: vision, olfaction, taste, audition, touch, proprioception, and vestibular sensations (Robertson and Simmons 2013). In each modality, three questions assess hypersensitivity, and three others hyposensitivity. The questionnaire evaluates how frequently some sensory events are experienced by the participant (for instance, "Do you find certain noises/pitches of sound annoying?"). Participants answer using the scale: never ( 0 point $)$, rarely (1 point), sometimes ( 2 points), often ( 3 points) or always ( 4 points). Total scores can range from 0 to 168 points.

\section{Translation of the Glasgow Sensory Questionnaire}

The GSQ was translated into French, after an agreement with the authors of the original English version (A. Robertson and D. Simmons), using the guidelines setup by the European Regulatory Issues on Quality of Life Assessment (ERIQA) group (Acquadro et al. 2008). This involved a translation/expert committee, which is now considered superior to the well-known back-translation procedure (Douglas and Craig 2007; Epstein et al. 2015) of four English/French bilinguals, with expertise in autism. Once a consensus was reached, the translation was tested using a focus group of six French native participants (3 neurotypicals and 3 with ASD), representatives of the target population, whose input prompted minor changes in the wording of item number 5. Participants were always encouraged to query if one item seemed not clear, but no requests were made and no missing answers were obtained for any of the items. The translated French language version is available in Appendix 1.

\section{Data analysis}

Scores calculation 
For each participant, we calculated the total AQ score (which can range from 0 to 50 ), and the five AQ subscores (which can range from 0 to 10 ) depicting social skills, attention to skills, attention to details, communication skills, and imagination skills. In addition, we calculated the GSQ score (which can range from 0 to 168), corresponding to the sum of the hypersensitivity and hyposensitivity scores (each ranging up to 84, and made of 21 items). We also extracted the subscores corresponding to each of the seven sensory modalities (each ranging up to 24), as well as the fourteen subscores corresponding to scores of hypersensitivity or hyposensitivity in each of the seven sensory modalities (each ranging up to 12). We calculated three coefficients of variation: one calculated across the seven sensory modalities, one across the 14 subscales (hypersensitivity and hyposensitivity scores of the seven sensory modalities), and one across the 42 items of the GSQ.

\section{Cross sample and cross language comparisons}

The scores obtained in the present study were compared to the scores obtained by Robertson \& Simmons (2013), and Ward et al. (2017) English populations, and to the scores obtained by Takayama et al. (2014) in a Japanese population. The mean scores and standard deviations obtained at each question of the GSQ from the original English version were correlated to our results using Pearson correlation test. Mean scores were compared between these two versions using one-way t-test comparing our samples to the mean obtained in the English version.

\section{Internal structure analysis}

Common factor analysis was performed on the 42 GSQ items for the entire population $(n=245)$, and on the 14 GSQ subscales of the low AQ and high AQ groups, using parallel analysis for number of factors extraction and oblique (oblimin) factor rotation. Separate analysis for both low AQ and high AQ group allowed to compare the internal structure of the 
GSQ in both groups and to identify underperforming items. For factor extraction, both the maximum likelihood and principal axis factoring methods were used (Beavers et al. 2013), but as they yielded very similar results, only the results pertaining to the maximum likelihood method are reported. In order to determine whether factors obtained on the 14 GSQ subscales reflect the same intended structure, hierarchical cluster analyses using Revelle's ICLUST algorithm (Revelle 1978, 1979) were performed. Revelle's method has been specifically designed to visualize questionnaire scales and subscales, based on two indices: alpha and beta coefficients. Alpha coefficient (mean split-half reliability) measures internal consistency, while Beta coefficient reflects factorial homogeneity (specifically, beta is the worst split-half reliability of a scale, and hence is lower than alpha). In short, the scale structure is built starting from two-item clusters showing the highest similarity between each other, and an item is added to the initial two-item cluster only if this addition improves the internal consistency (measured by alpha) and/or the factorial homogeneity (measured by beta) of the cluster. These results are given in a hierarchical tree diagram of clusters that displays the internal sub-structure of the scale, allowing the definition of homogeneous subscales. The tree diagram connects increasingly less similar items and/or clusters from left to right: the most similar items are combined first, and the most dissimilar items are added last. Alpha and beta coefficients are provided for each cluster, and correlation coefficients are given for each connection. Alpha above .8 and beta above .7 , with differences between them lower than .1 are considered good signs of homogeneity and internal consistency (Cooksey 2006). An index of goodness of fit allows a comparison of the quality of the structures. The fit of the different models to the data was checked by the RMSR method (root mean square residuals), where a value below .05 is considered a good fit, and values between .05 and .08 a fair fit (Cooksey 2006; Fabrigar et al. 1999). This technique is considered as very complementary to the more classical approach of factor analysis and is less method dependent (Cooksey 2006). Factor, 
cluster and internal validity measures were performed using the "Psych" package version 1.6.9 (Revelle, 2008) within the R statistical package version 3.3.1 (June 2016).

\section{Structural validity assessment}

Correlations between the AQ and GSQ scores were performed using Pearson correlation analysis. When multiple comparisons were performed, the p-value was corrected by the number of comparisons using Bonferroni correction. Hence, for correlations involving the five subscales of the AQ scores, the significance threshold was set at $p_{\text {corrected }}<.01$. For correlations involving the seven subscales of the GSQ scores, the significance threshold was set at $p_{\text {corrected }}<.007$.

\section{Group comparisons}

Comparisons of the total AQ or GSQ scores between the low and high AQ groups were examined using Student's t tests. The differences in subscores of the GSQ were studied using three-way mixed ANOVA with factor group (two levels, inter-subjects), sensory modality (seven levels, intra-subjects) and hyper/hyposensitivity aspects (two levels, intrasubjects). Within groups, hyper and hyposensitivity scores were compared using paired Student t-tests. Correlation matrices were compared using Steiger test (Steiger 1980) (implemented in Revelle's Psych $^{\circ}$ package). Correlation coefficients were compared between groups using Fisher r-to-z transformation.

All statistical analyses were performed using R (http://www.r-project.org/, version 3.3.1.) and the threshold for statistical significance was set at $p<.05$, and was corrected for multiple comparisons using Bonferroni correction. 


\section{Results}

\section{Validation of the French version of the GSQ}

\section{Comparisons across samples and languages}

Both the mean AQ and GSQ in the present study were very close to the ones found in the English version (Robertson and Simmons 2013): mean AQ of $23.0( \pm 13.8)$ in the French version and of $22.5( \pm 10.6)$ in the English version, mean GSQ of $58.7( \pm 28.4)$ in the French version and of $56.7( \pm 23.6)$ in the English version. The mean scores of each of the 42 questions were highly positively correlated between the English and the French versions $(r=$ $.83, p<10^{-6}$ ) (Figure 1.A). Moreover, for each question, the mean score in the French version was at less than one standard deviation from the mean of the English version, except for question $n^{\circ} 39$ (French study: score $=1.4 \pm 1.3$, English study: score $=0.3 \pm 0.7, t=14, p<10^{-}$ ${ }^{6}$ ) (Figure 1.B). The standard deviations of the scores of the GSQ questions were positively correlated between the French and English versions $\left(r=.63, p<10^{-5}\right)$. Finally, the correlation coefficients between the AQ and the GSQ were also very similar in the two studies (French study: $r=.81 ;$ English study: $r=.78)$.

The 14 GSQ subscores (hyper- or hyposensitivity scores across the seven sensory modalities) were highly correlated in our dataset and in the dataset by Ward and colleagues (Ward et al. 2017). Indeed, there was a strong correlation between the scores they got in their control group (whose mean AQ was 19) and the scores from our group of participants with low AQ $\left(r=.90, p<10^{-4}\right)$. In addition, there was a strong correlation between the scores of their ASD group and of our high AQ group $\left(r=.96, p<10^{-4}\right)$. 
The GSQ subscores from the seven sensory modalities obtained in our low AQ group correlated with the subscores from the control groups in the English (Ward et al. 2017) $(r=$ $.93, p<.01)$ and Japanese (Takayama et al. 2014) $(r=.82, p<.05)$ GSQ studies.

\section{[Please, insert Figure 1]}

\section{Internal reliability}

Cronbach's alpha, which assesses reliability, was equal to .95 for the all 42 items and to .94 for the 14 subscales. Per group, it was equal to .86 in the low AQ group and to .93 in the high AQ group for the 42 items. For the 14 subscales, it was equal to .84 in the low AQ group and to .91 in the high AQ group.

Item to total correlations was .59 on average, with the lowest correlations for two questions assessing olfactory hyposensitivity (question $n^{\circ} 36(.18)$ and $n^{\circ} 17(.33)$ ). It was equal to .38 in the low AQ group and to .50 in the high AQ group. The details of those correlations are given in Appendix 2 (Table SI-B).

\section{Factor analysis of the internal structure (42 questions, 245 subjects)}

Prior to factor analysis, ten multivariate outliers found using Mahalanobis distance with $p<10^{-3}$ were excluded, leaving 235 cases, with a ratio of subjects per variable of 5.6.

The Kaiser-Meyer-Olkin measure of sampling adequacy (KMO) was .94 and all values for individual items were greater than .84 , except for two items assessing olfactory hyposensitivity (items $n^{\circ} 36: .58$ and $n^{\circ} 7: .78$ ). Bartlett's test was highly significant $\left(\chi^{2}>5162\right.$, $p<10^{-4}$ ), confirming that correlations between individual items were sufficient.

The maximum likelihood method was used for factor extraction. Two factors had an eigenvalue greater than 1 , with percentages of variance explained of $36 \%$ and $3 \%$. Inspection of Cattell's scree plot, parallel analysis and Velicer's MAP criterion (Velicer 1976) gave two factors. The fit of the model to the data was good, based on a RMSR result of .03, where a 
value below .05 is considered a good fit (Fabrigar et al. 1999; Field et al. 2012). The residuals were normal (Kolmogorov-Smirnov test $=.02, p>.05)$. The Tucker Lewis Index of factoring reliability was .84 and the comparative fit index (CFI) was .86 , which is in the lower range of acceptable fits. The average communalities obtained across the 42 items was .39 ( \pm .13$)$, with seven items below .30. The lowest communalities were obtained for three items investigating the hyposensitivity olfactory modality $\left(\mathrm{n}^{\circ} \mathrm{7}, 17\right.$ and 36$)$.

Oblique rotation showed two underlying factors, explaining respectively $25 \%$ and $14 \%$ of variance. The correlation between these two factors was .62. The individual loadings of each factor on the 42 items are given in Appendix 2 (Table SI-C) and are summarized here.

The loadings on Factor 1 involved 25 items, including 19 items assessing hypersensitivity. The highest loads $(>.60)$ were obtained for ten hypersensitivity items.

The loadings on Factor 2 involved 16 items including 12 items with loads ranging from .41 to .71 , all assessing hyposensitivity. Among these items, six showed a high degree of crossload between both factors (one gustatory, three proprioceptive and two visual). One olfactory hyposensitivity item $\left(\mathrm{n}^{\circ} 7\right)$ did not load sufficiently on any factor.

Although not every item is loading clearly on one single factor, the two-factor solution is in agreement with Factor 1 regrouping mostly hypersensitivity items and Factor 2 regrouping hyposensitivity items.

\section{[Please, insert Figure 2]}

\section{Relationship between the GSQ sensory score and the AQ}

The AQ scores ranged from 1 to $49($ mean $=23.0 \pm 13.8)$, and the GSQ scores ranged from 12 to $144($ mean $=58.7 \pm 28.4)($ Table 1$)$. The GSQ and AQ were highly positively correlated $\left(r=.81, p<10^{-6}\right.$, Figure 2.A). The subscales of the AQ positively correlated with the total GSQ score. The GSQ subscales also correlated with the total AQ (Table 2). Finally, 
the hypersensitivity and hyposensitivity scores of the GSQ were strongly correlated $(r=.82, p$ $<10^{-6}$, Figure 2.B) in total, and for each sensory modality (Table 3 ).

\section{[Please, insert Table 2]}

\section{Group comparison: low $A Q$ versus high $A Q$ groups}

The demographic data of the two groups and basic descriptive statistics are given in Table 1. The average AQ of the low AQ group was $12.4( \pm 5.1)$, while the high AQ group scored $38.0( \pm 6.0)$ in average (group difference: $\left.t=35, p<10^{-6}\right)$. The GSQ was significantly higher in the high AQ group than in the low AQ group for the total score $(82.8 \pm 25.4 \mathrm{vs} .41 .6$ $\left.\pm 14.7, t=15, p<10^{-6}\right)$, the hypersensitivity score $\left(46.9 \pm 14.9\right.$ vs. $\left.21.9 \pm 8.4, t=15, p<10^{-6}\right)$, and the hyposensitivity score $\left(35.9 \pm 12.4\right.$ vs. $\left.19.6 \pm 8.0, t=12, p<10^{-6}\right)$ (Figure 3.A).

\section{[Please, insert Figure 3]}

\section{Correlation between the $A Q$ and GSQ scores}

The GSQ and AQ scores were positively correlated in both groups (low AQ: $r=.45, p$ $<10^{-6}$, high AQ: $r=.62, p<10^{-6}$, Figure $2 . A$ ), with a significantly different linear relationship between the two groups. The relationship between the GSQ score and AQ writes as follows: $[\mathrm{GSQ}=1.3 * \mathrm{AQ}+25.5]$ in the low AQ group, and [GSQ $\left.=2.6^{*} \mathrm{AQ}-16.0\right]$ in the high AQ group. The $95 \%$ confidence intervals of the slopes were $[0.87,1.7]$ in the low AQ group, and $[1.9,3.3]$ in the high AQ group. The $95 \%$ confidence intervals of the intercepts were $[19.7$, 31.2] in the low AQ group and $[-41.4,9.3]$ in the high AQ group. Hence, the 95\% confidence intervals of both slopes and intercepts did not overlap between groups.

The correlations between the subscales of the GSQ and of the AQ are described in Table 2. Fisher's z' transformations showed that correlation coefficients were significantly greater in the high $\mathrm{AQ}$ group than in the low $\mathrm{AQ}$ group for the correlation between the $\mathrm{AQ}$ and the hypersensitivity GSQ score $(r=.61$ vs. $r=.37, t=2, p<.01)$. Moreover, in the high 
AQ group, correlation coefficients were also stronger for the correlation between the AQ and the olfactory GSQ subscore $(r=.45$ vs. $r=.12, t=3, p<.01)$ and the tactile GSQ subscore $(r$ $=.30$ vs. $r=.54, t=2, p<.05)$ than the low AQ group. A stronger correlation in the high AQ group was also found between the total GSQ score and the communication AQ subscore ( $r=$ .52 vs. $r=.28, t=2, p<.05)$.

\section{[Please, insert Table 3]}

\section{GSQ hypersensitivity and hyposensitivity scores}

The hypersensitivity and hyposensitivity scores of the GSQ were highly positively correlated within groups (low AQ: $r=.60, p<10^{-6}$; high AQ: $r=.73, p<10^{-6}$; Figure 2.B, Table 3). Significantly greater correlation coefficients were found in the high AQ group as compared to the low AQ group for the correlations between the hypersensitivity and hyposensitivity scores for the visual $(r=.54$ vs. $r=.31, t=2, p<.05)$ and the vestibular $(r=$ .46 vs. $r=.18, t=2, p<.01)$ modalities, and a marginally significant difference was found for the gustatory modality ( $r=.45$ vs. $r=.23, t=2, p=.06)$.

The ANOVA investigating the effect of group ( 2 levels), sensory modality ( 7 levels) and hyper or hyposensitivity (2 levels) on scores, revealed a group effect $(F(1,243)=255, p<$ $\left.10^{-6}\right)$, a sensory modality effect $\left(F(6,1458)=175, p<10^{-6}\right)$, and a hyper/hyposensitivity effect $\left(F(1,243)=115, p<10^{-6}\right)($ Figure 3). There was an interaction between group and sensory modality $\left(F(6,1458)=11, p<10^{-6}\right)$, and hyper/hyposensitivity $\left(F(1,243)=61, p<10^{-6}\right)$. Finally, there was a triple interaction between group, sensory modality and hyper/hyposensitivity $\left(F(6,1458)=9, p<10^{-6}\right)$. In both groups, the GSQ scores were higher for hypersensitivity than hyposensitivity: $21.9( \pm 8.4)$ versus $19.6( \pm 8.0)\left(t=4, \mathrm{p}<10^{-6}\right)$ in the low AQ group, and $46.9( \pm 14.9)$ versus $35.9( \pm 12.4)\left(t=11, p<10^{-6}\right)$ in the high AQ group (Figure 3.A). 
The high AQ group scored higher than the low AQ group at every subscale $\left(p<10^{-6}\right.$ for every modality, Figure 3.B).

Within group, both the low AQ and high AQ groups reported significantly higher hypersensitivity than hyposensitivity for the visual, auditory and vestibular modalities (Figure 3.B). Both groups showed higher hyposensitivity than hypersensitivity for the proprioceptive modality. Contrary to the low AQ group, the high AQ group also showed significantly higher scores in hypersensitivity than hyposensitivity for the gustatory, olfactory and tactile modalities.

The difference between the hypersensitivity score and the hyposensitivity score was significantly greater in the high AQ group than in the low AQ group (11.0 \pm 10.2 vs. $2.3 \pm 7.3$, $t=6, p<10^{-6}$ ). In order to estimate the relative weight of the hyper and hyposensitivity scores, we divided the hypersensitivity score by the hyper plus hyposensitivity scores in each sensory modality. Results showed higher relative hypersensitivity in the high AQ group as compared to the low AQ group for the gustatory ( $54 \%$ vs. $47 \%, t=3, p<.01)$, olfactory ( $64 \%$ vs. $\left.47 \%, t=6, p<10^{-6}\right)$ and proprioceptive $(45 \%$ vs. $35 \%, t=3, p<.01)$ modalities (these pvalues are all under the .007 threshold with Bonferroni correction).

\section{Correlation matrices between the GSQ subscores}

Correlation matrices between the 42 items of the GSQ showed greater correlations for the high AQ group than the low AQ group $\left(\chi^{2}=1803, p<10^{-6}\right)$, especially between items representing the same modalities. Similar differences in correlations between the 14 subscales (hyposensitivity and hypersensitivity in the seven sensory modalities) were observed, with significantly greater correlations within the high AQ group than within the low AQ group $\left(\chi^{2}\right.$ $=220, p<10^{-6}$ ). Between groups, no significant differences were observed in the correlation matrices obtained with the hyposensitivity subscores $\left(\chi^{2}=28, p>.05\right)$. However, highly 
significant group differences were obtained for hypersensitivity subscores $\left(\chi^{2}=85, p<10^{-6}\right.$, Figure 4).

As these stronger correlations suggest greater consistency of the high AQ group responses, it led us to analyze the variation coefficient calculated for each subject across the 14 subscales. The analysis of variance showed a significantly greater variation coefficient in the low AQ group $(64 \% \pm 19)$ than in the high AQ group $(46 \% \pm 19)\left(F(1,239)=50, p<10^{-6}\right)$. The same differences between groups were obtained whether the variation coefficients were calculated across the seven hypersensitivity subscales, the seven hyposensitivity subscales, the 14 subscales or the 42 items $\left(105 \% \pm 27\right.$ vs. $\left.70 \% \pm 27 ; F(1,239)=101, p<10^{-6}\right)$. This variation coefficient was negatively correlated with the GSQ score $\left(r=-.78, p<10^{-6}\right)$ and with the AQ score $\left(r=-.52, p<10^{-6}\right)$. In other words, in the high AQ group, subjects not only had greater GSQ scores but also tended to be more consistent in their answers.

\section{[Please, insert Figure 4]}

Factor analysis on the 14 subscales in the low AQ and high AQ groups:

As one of the advantages of the GSQ is to be able to test both hyposensitivity and hypersensitivity on seven sensory modalities, we proceeded with a factor analysis on the 14 subscales. This allowed to maintain a good subject to variable ratio (i.e., $>7$ ) whilst being able to perform it separately on the two AQ groups: as the correlation matrices of both groups showed significant different patterns of correlations and significantly greater correlations in the high AQ than in the low AQ group, investigating a potential different internal structure between both groups seemed quite relevant.

The number of multivariate outliers excluded was 0 for the high AQ subjects and 2 for the low AQ subjects, so the factor analyses were performed on the 102 subjects with high AQ and 141 subjects with low AQ. 
Skewness ranged between -.9 and .7 in the high AQ group and between .1 and 1.4 in the low AQ group. Kurtosis was below 3, except for two variables below 3.6 in the high AQ group, and six variables below 4.7 in the low AQ group. The KMO was very good (high AQ: .89, low AQ: .86), and all values for individual items were greater than .82 (olfactory hyposensitivity) in the high AQ group, and greater than .76 (auditory hypersensitivity) in the low AQ group. Bartlett's test was highly statistically significant (high AQ: $\chi^{2}>660, p<10^{-4}$; low AQ: $\chi^{2}>524, p<10^{-4}$ ), confirming that correlations between individual items were sufficient.

The maximum likelihood method was used for factors extraction. Inspection of Cattell's scree plot gave a number of factors of one or two in the high AQ group and of two in the low AQ group. Parallel analysis gave one factor in the high AQ group, and two factors in the low AQ group. Finally, Velicer's MAP criterion gave two factors in both groups. For both groups, only one factor had an eigenvalue greater than 1 . Hence, we tested the option with either one factor or two factors.

The two factors explained $48 \%$ of variance in the high AQ group (43\% and $5 \%)$ and $36 \%$ of variance in the low AQ group (30\% and $6 \%$ ). The model fit was good based on a RMSR result of .06 in the high AQ group and .05 in the low AQ group. The residuals were normal in both groups ( $p>.05$ in both groups). The Tucker Lewis Index of factoring reliability was .94 in the high AQ group and .97 in the low AQ group. There were very good CFI: .96 in the high AQ group and .98 in the low AQ group.

The one-factor option explained $43 \%$ of the variance in the high AQ group and $30 \%$ of variance in the low AQ group. It gave a model with lower fits indexes: Root Mean Square Error of Approximation of .09 in the high AQ group and .07 in the low AQ group, RMSR of .07 in the high AQ group and .08 in the low AQ group, Tucker Levis $<.90$ in both groups and CFI at .91 in both groups. Hence the two-factor extraction was selected for both groups. 
The average communality obtained across the 14 variables was $.48( \pm .1)$ in the high AQ group and $.36( \pm .09)$ in the low AQ group. In the high AQ group, they were all above .45, except for the olfactory hyposensitivity score at .18 and the auditory hyposensitivity score at 0.38. In the low AQ group, all communalities were between .21 and .51 .

Oblique rotation showed two underlying factors, explaining respectively $25 \%$ and $24 \%$ of variance in the high AQ group, and $20 \%$ and $16 \%$ of variance in the low AQ group. The correlation between the two factors was .66 in the high AQ group and 0.53 in the low AQ group. The individual loadings of each factor on each of the 14 variables (Appendix 2, Table SI-D) are summarized below.

\section{High AQ group}

In the high AQ group, the seven hypersensitivity scores loaded on Factor 1, with some degrees of cross-loadings for the proprioceptive, visual and vestibular modalities. The seven hyposensitivity scores loaded on Factor 2, with a low load for the olfactory hyposensitivity score and the highest load for the tactile hyposensitivity score (.84).

Hierarchical cluster analysis revealed a homogeneous main cluster $(\mathrm{C} 12$, with $\alpha=.91$, $\beta=.84)$ and two main subclusters: one associated with hypersensitivity variables $(\mathrm{C} 11$, with $\alpha$ $=.88, \beta=.82$ ) and one associated with hyposensitivity variables (except for the olfactory hyposensitivity variable) $(\mathrm{C} 9, \alpha=.85, \beta=.80)$ (Figure 5). However, the addition of the olfactory hyposensitivity variable led to a drop in homogeneity $(\beta=.6)$, showing that this variable did not fit well. Likewise, when we force the number of final clusters to be 2 , the olfactory hyposensitivity variable was excluded as a single item cluster, while the rest of the structure was identical to the previous main cluster (C12) with two hyposensitivity and hypersensitivity subclusters. For the entire structure (excluding the olfactory hyposensitivity variable), the minimum $\alpha$ and $\beta$ were .66 and above .80 for the main subclusters, with a 
difference between them lower than .08. This result argues for the unidimensionality of the structure, with two subclusters corresponding to hyposensitivity and hypersensitivity.

\section{[Please, insert Figure 5]}

\section{Low AQ group}

In the low AQ group, the seven hyposensitivity variables and the proprioceptive hypersensitivity variable loaded on Factor 1. The six remaining hypersensitivity variables loaded on Factor 2. There was some cross-loading with the gustatory hypersensitivity and auditory hyposensitivity variables, and to a lesser degree, with the visual hypersensitivity variable.

Cluster analysis performed on the low AQ group revealed a somewhat foggier pattern than for the high AQ group (Appendix 2, Figure SI-A), with lower $\alpha$ and $\beta$ coefficients (mostly ranging between .6 and .7) and more heterogeneity (Supplementary information SI.3). A main cluster was clearly identified $(\mathrm{C} 11$, with $\alpha=.84, \beta=.74)$, regrouping two main subclusters, corresponding to hyposensitivity $(\mathrm{C} 10$, with $\alpha=.80, \beta=.71)$ and hypersensitivity (C8, with $\alpha=.72, \beta=.69$ ). The proprioceptive hypersensitivity variable was associated with the vestibular hyposensitivity variable, and was part of the hyposensitivity cluster, as in the factor analysis. The addition of the olfactory hyposensitivity and auditory hypersensitivity variables yielded a drop in beta coefficient (i.e. a loss of homogeneity), suggesting that those two items did not fit well and had a somewhat different behaviour than the rest of the scale.

\section{Discussion}

This study aimed at validating a French language version of the Glasgow Sensory Questionnaire and at refining the characterization of sensory sensitivity profiles in individuals 
with low and high AQ. Our results validate the French-language version of the GSQ, within the framework of the equivalences defined by the Universalist Model of cross-cultural adaptation of health questionnaires (Herdman et al. 1998). Overall, this study confirms the strong relationship between the number of autistic traits and the frequency of unusual sensory experiences. We also defined precise sensory profiles of hypersensitivity and hyposensitivity across sensory modalities in individuals with low and high AQ, and we characterized group differences.

\section{Validation of the French version of the GSQ}

The strong correlations obtained across the 42 items between the French and English (Robertson and Simmons 2013) studies are in favor of good item equivalence between languages. Item equivalence between population samples was also respected as scores at the fourteen subscales of the present study and of the study by Ward and colleagues (2017) strongly correlated. The French GSQ showed a very good internal consistency, in line with the ones reported in the English and Japanese versions of the GSQ (Robertson and Simmons 2013; Takayama et al. 2014). Functional equivalence, dealing with the relationships between the questionnaire and external factors, was also respected as correlations between GSQ and AQ subscales were very similar across languages and samples (Robertson and Simmons 2013; Takayama et al. 2014).

The internal structure of the GSQ had not been investigated in previous studies, but was characterized in the present study in order to determine whether it reflected the expected structure of the questionnaire. Precisely, we expected the factor analysis to reveal seven factors corresponding to the seven sensory modalities, and/or two factors corresponding to hyposensitivity and hypersensitivity, or a mix of the two. Common factor analysis performed on the entire population, yielded a two-factor structure with one factor encompassing mostly hypersensitivity items, and the other involving mostly hyposensitivity items. Hierarchical 
cluster analysis confirmed this structure in both AQ groups, showing a unique dimension scale ("sensory abnormality"), with two main subcomponents: hyper and hyposensitivity. However, finer grain analysis reveals differences between low and high AQ groups.

\section{Refining sensory profiles in groups with low versus high $A Q$}

GSQ scores increased twice more with the AQ for the high AQ group than for the low AQ group. The non-linear relationship between AQ and GSQ scores across the entire AQ range highlighted the fact that groups with low and high AQ had distinct sensory profiles. Indeed, it suggests that individuals with high AQ are not simply characterized by an increased occurrence of atypical sensory experiences, but possibly also by different sensory profiles.

Sensory profiles in groups with low and high AQ were first analyzed using cluster and factor analyses on the fourteen GSQ subscales. Factor analysis showed a similar two-factor structure (hypersensitivity and hyposensitivity) in each group, but with more variance being explained for the high AQ than for the low AQ group. Cluster analysis showed a similar structure at a macro scale, i.e., an abnormal sensitivity factor with two subcomponents, hypersensitivity and hyposensitivity with a clearer pattern in the high AQ group, and a foggier pattern in the low AQ group. Finer grain analysis shows a slightly different relationship between the items, with a stronger homogeneity of the two subcomponents in the high AQ group versus the low AQ group. The hypo-olfactory item is clearly a "rogue" item for both groups (which is in agreement with the behavior of the corresponding items in the 42 items scale). However, cluster analysis allows to pin-point differences in how the "hyper-auditory" item behaves: it is associated with the hyper-vestibular item in the high AQ group, but behaves as an outsider for the low AQ group. In future development of sensory analysis, the differential perception of such items between the low AQ group and the high AQ group could be worth investigating. In addition, the analysis of the GSQ structure also demonstrated a greater consistency between items in the high AQ group. 
The difference between groups is further illustrated by the correlations between the hypersensitivity and hyposensitivity scores in the seven sensory modalities. Indeed, the correlation matrices showed stronger correlations in the high AQ than in the low AQ group, especially for the hypersensitivity subscales. This indicates that individuals with high AQ have an "extreme" sensitivity, shared quite uniformly across the seven sensory modalities. In contrast, it suggests that individuals with low AQ would have "peaks" of increased/decreased sensitivity in a particular sensory modality, but this would not be generalized to every sensory modality. Together with the clear and robust patterns revealed by the cluster and common factor analyses, these elements show that the high AQ group had a more homogeneous profile of sensory sensitivity than the low AQ group. Interestingly, the high AQ group also showed stronger correlations between hyposensitivity and hypersensitivity scores, especially in the visual modality. It means that, contrary to people with low AQ, individuals with high AQ have a higher tendency to experience both hypersensitivity and hyposensitivity within a sensory modality.

The high AQ group scored above the low AQ group for each of the fourteen subscales, except for the olfactory hyposensitivity subscale. There was no difference either on this subscale in the comparison between control and ASD individuals in the study by Ward and colleagues (Ward et al. 2017). In our study, the two groups were also distinguished by the fact that the high AQ group reported more experiences due to hypersensitivity than hyposensitivity, as compared to the low AQ group. The relative predominance of hypersensitivity over hyposensitivity was more pronounced in the high AQ than low AQ group for the olfactory modality, and to a lower extent, for the gustatory and proprioceptive modalities. Noticeably, enhanced olfactory hypersensitivity had been previously linked to more autistic traits (Ashwin et al. 2014). 


\section{Linking sensory profiles of people with high AQ to current theories of ASD}

Our results showed quite homogeneous profiles of sensory sensitivity in the high AQ group, and sensory profiles characterized by peaks of hypersensitivity or hyposensitivity in different sensory modalities in the low AQ group. Yet, given the notion of sensory expertise in ASD, we could have expected sensory profiles with peaks of hypersensitivity more pronounced in the high AQ group than in the low AQ group. The stronger predominance of experiences of hypersensitivity over hyposensitivity in the high AQ group could contribute to explain why theories of ASD were often focused on hypersensitivity. The EPF theory (Mottron et al. 2006; Mottron, and Burack 2001) or the low endogenous noise theory of ASD (Davis and Plaisted-Grant 2015) would be consistent with the frequent hypersensitivity experiences in ASD. To explain the co-occurrence of both hyposensitivity and hypersensitivity within a sensory modality in ASD, the global context needs to be taken into account. For instance, the predictable nature of the sensory stimulations seems to play a key role in the reactivity to sensory stimuli in people with ASD (Ashburner et al. 2013). Indeed, a study based on questionnaires revealed that around half of the variance of sensory sensitivity abnormalities in ASD could be explained by anxiety and intolerance to uncertainty (Neil et al. 2016). This need for predictive stimuli fits the Bayesian brain theories suggesting that ASD individuals would have suboptimal predictive systems, particularly in changing environments (Haker et al. 2016; Lawson et al. 2014; Palmer et al. 2017; Robic et al. 2014; Van de Cruys et al. 2014, 2016). Bayesian theories could explain the presence of both hypersensitivity and hyposensitivity, as sensory reactivity would depend on the context (and particularly, of its predictability). The context should help disentangling sensory relevant information from irrelevant noise, and therefore adapt the level of precision of the sensory input (Van de Cruys et al. 2016). Hence, depending on the context, noisy sensory inputs would lead to hyposensitivity, while precise sensory inputs would lead to hypersensitivity. 


\section{Implications for individuals with ASD}

As abnormal sensory sensitivity can affect the quality of life, a finer characterization of the sensory sensitivity profiles could help defining better working and living environments for individuals with high AQ or diagnosed with ASD. Based on ASD reports, Smith and Sharp (2013) suggested that heightened senses in ASD would lead to fascination and pleasurable activities, but would also trigger stress, avoidance and isolation. Atypical sensitivity can affect non-social, but also social perception. For instance, Lundqvist (2015) showed that hyper-responsiveness to touch mediated social dysfunction in ASD. For people with ASD, the major negative outcomes of hypersensitivity appear to be over-responsivity and anxiety. Indeed, sensory over-responsivity positively correlates with the AQ (Tavassoli et al. 2014) and can lead to anxiety (Ashburner et al. 2013). Anxiety disorders are more common in individuals with ASD than controls, and are closely related to sensory over-responsivity (Green and Ben-Sasson 2010). Moreover, in children with ASD, sensory over-responsivity would emerge earlier than anxiety (Green et al. 2011), and this atypical sensitivity leads to higher physiological stress (Corbett et al. 2016). Interestingly, Horder et al. (2014) showed that the correlation between the GSQ and the AQ survived correction for anxiety trait scores. To cope with atypical sensory experiences, people with ASD would use several strategies such as avoidance, meta-cognitive adaptation, or increasing the predictability of the environment (Ashburner et al. 2013).

\section{Perspectives}

With the GSQ internal structure analysis, the present work suggests that new GSQ indices could be relevant for exploring the peculiarities of perceptual processing in ASD. To allow further cross samples and cross languages comparisons, we provided detailed analyses in Appendix 2. Replicating this study on a different high AQ population would allow ascertaining the robustness of the associations between different sensory modalities observed 
here. Indeed, the robustness of those similarities should be assessed in other samples so as to determine whether these similarities are specific to ASD. Indeed, heightened hypersensitivity and hyposensitivity was also found in synaesthetes (Ward et al. 2017), and comparing the GSQ internal structure of the responses of synaesthetes and of subjects with ASD could indicate whether they show different sensory modalities associations.

Finally, future works should investigate the relationships between the GSQ scores and behavioral measures (such as perceptual thresholds) or neurophysiological data in order to estimate whether the self-report of the GSQ fits physiological data. The GSQ is designed to assess sensory sensibility in individuals with ASD who are able to complete a self-report questionnaire, hence limiting its scope to only part of the spectrum. Future works could also adapt this self-report questionnaire to an observation measurement tool for a larger use.

In conclusion, this study confirms the robust link between autistic traits and atypical sensory sensitivity. Although the GSQ is relatively recent, the similarities of behavior observed between a French sample and several other samples, with good conceptual, measurement and functional equivalences, are extremely encouraging to go toward an international standard use of the GSQ in clinical and research contexts.

\section{Compliance with Ethical Standards}

Funding: This study was supported by a Scientific Research Council grant from the Vinatier Hospital Center, and was performed within the framework of the LABEX CORTEX (ANR11-LABX-0042) of Université de Lyon, within the program "Investissements d'Avenir" (ANR-11-IDEX-0007) operated by the French National Research Agency (ANR).

Ethical approval: All procedures performed in this study were in accordance with the standards of the French ethical guidelines and with the 1964 Helsinki declaration and its later 
amendments or comparable ethical standards. Informed consent was obtained from all individual participants included in this study.

\section{References}

Acquadro, C., Conway, K., Hareendran, A., Aaronson, N., \& European Regulatory Issues and Quality of Life Assessment (ERIQA) Group. (2008). Literature review of methods to translate health-related quality of life questionnaires for use in multinational clinical trials. Value in Health: The Journal of the International Society for Pharmacoeconomics and Outcomes Research, 11(3), 509-521. doi:10.1111/j.15244733.2007.00292.x

American Psychiatric Association. (2013). Diagnostic and Statistical Manual of Mental Disorders. American Psychiatric Association. http://dsm.psychiatryonline.org/doi/book/10.1176/appi.books.9780890425596.

Ashburner, J., Bennett, L., Rodger, S., \& Ziviani, J. (2013). Understanding the sensory experiences of young people with autism spectrum disorder: a preliminary investigation. Australian Occupational Therapy Journal, 60(3), 171-180. doi:10.1111/1440-1630.12025

Ashwin, C., Chapman, E., Howells, J., Rhydderch, D., Walker, I., \& Baron-Cohen, S. (2014). Enhanced olfactory sensitivity in autism spectrum conditions. Molecular Autism, 5(1), 53. doi:10.1186/2040-2392-5-53

Asperger, Hans. (1944). Die 'Autistischen Psychopathen' im Kindesalter. Archiv für Psychiatrie und Nervenkrankheim, 117, 76-136.

Baranek, G. T., David, F. J., Poe, M. D., Stone, W. L., \& Watson, L. R. (2006). Sensory Experiences Questionnaire: discriminating sensory features in young children with autism, developmental delays, and typical development. Journal of Child Psychology and Psychiatry, 47(6), 591-601. doi:10.1111/j.1469-7610.2005.01546.x

Baron-Cohen, S., Wheelwright, S., Skinner, R., Martin, J., \& Clubley, E. (2001). The autismspectrum quotient (AQ): evidence from Asperger syndrome/high-functioning autism, males and females, scientists and mathematicians. Journal of Autism and Developmental Disorders, 31(1), 5-17.

Baum, S. H., Stevenson, R. A., \& Wallace, M. T. (2015). Behavioral, perceptual, and neural alterations in sensory and multisensory function in autism spectrum disorder. Progress in Neurobiology, 134, 140-160. doi:10.1016/j.pneurobio.2015.09.007

Beavers, A. S., Lounsbury, J. W., Richards, J. K., Huck, S. W., Skolits, G. J., \& Esquivel, S. L. (2013). Practical Considerations for Using Exploratory Factor Analysis in Educational Research. Practical Assessment, Research \& Evaluation, 18(6). Accessed 23 September 2017

Bennetto, L., Kuschner, E. S., \& Hyman, S. L. (2007). Olfaction and taste processing in autism. Biological Psychiatry, 62(9), 1015-1021. doi:10.1016/j.biopsych.2007.04.019 
Blakemore, S.-J., Tavassoli, T., Calò, S., Thomas, R. M., Catmur, C., Frith, U., \& Haggard, P. (2006). Tactile sensitivity in Asperger syndrome. Brain and cognition, 61(1), 5-13. doi:10.1016/j.bandc.2005.12.013

Brock, J. (2012). Alternative Bayesian accounts of autistic perception: comment on Pellicano and Burr. Trends in Cognitive Sciences, 16(12), 573-574. doi:10.1016/j.tics.2012.10.005

Brown, C, \& Dunn, W. (2002). Adult/adolescent sensory profile: user's manual.

Cascio, C., McGlone, F., Folger, S., Tannan, V., Baranek, G., Pelphrey, K. A., \& Essick, G. (2008). Tactile Perception in Adults with Autism: a Multidimensional Psychophysical Study. Journal of autism and developmental disorders, 38(1), 127-137. doi:10.1007/s10803-007-0370-8

Cooksey, R. W. (2006). Coefficient Beta and Hierarchical Item Clustering: An Analytical Procedure for Establishing and Displaying the Dimensionality and Homogeneity of Summated Scales. Organizational Research Methods, 9(1), 78-98. doi:10.1177/1094428105283939

Corbett, B. A., Muscatello, R. A., \& Blain, S. D. (2016). Impact of Sensory Sensitivity on Physiological Stress Response and Novel Peer Interaction in Children with and without Autism Spectrum Disorder. Frontiers in Neuroscience, 10, 278. doi:10.3389/fnins.2016.00278

Crane, L., Goddard, L., \& Pring, L. (2009). Sensory processing in adults with autism spectrum disorders. Autism, 13(3), 215-228. doi:10.1177/1362361309103794

Dakin, S., \& Frith, U. (2005). Vagaries of visual perception in autism. Neuron, 48(3), 497507. doi:10.1016/j.neuron.2005.10.018

Davis, G., \& Plaisted-Grant, K. (2015). Low endogenous neural noise in autism. Autism: The International Journal of Research and Practice, 19(3), 351-362. doi:10.1177/1362361314552198

Douglas, S. P., \& Craig, C. S. (2007). Collaborative and Iterative Translation: An Alternative Approach to Back Translation. Journal of International Marketing, 15(1), 30-43. doi:10.1509/jimk.15.1.030

Dudova, I., Vodicka, J., Havlovicova, M., Sedlacek, Z., Urbanek, T., \& Hrdlicka, M. (2011). Odor detection threshold, but not odor identification, is impaired in children with autism. European Child \& Adolescent Psychiatry, 20(7), 333-340. doi:10.1007/s00787-011-0177-1

Epstein, J., Osborne, R. H., Elsworth, G. R., Beaton, D. E., \& Guillemin, F. (2015). Crosscultural adaptation of the Health Education Impact Questionnaire: experimental study showed expert committee, not back-translation, added value. Journal of Clinical Epidemiology, 68(4), 360-369. doi:10.1016/j.jclinepi.2013.07.013

Fabrigar, L. R., Wegener, D. T., MacCallum, R. C., \& Strahan, E. J. (1999). Evaluating the use of exploratory factor analysis in psychological research. Psychological Methods, 4(3), 272-299. doi:10.1037/1082-989X.4.3.272

Field, Andy, Miles, Jeremy, \& Field, Zoë. (2012). Discovering Statistics Using $R$ (SAGE Publications Ltd.). 
Foxe, J. J., Molholm, S., Del Bene, V. A., Frey, H.-P., Russo, N. N., Blanco, D., et al. (2015). Severe multisensory speech integration deficits in high-functioning school-aged children with Autism Spectrum Disorder (ASD) and their resolution during early adolescence. Cerebral Cortex (New York, N.Y.: 1991), 25(2), 298-312. doi:10.1093/cercor/bht213

Frith, U. (1991). Autism and Asperger Syndrome. Cambridge University Press.

Green, S. A., \& Ben-Sasson, A. (2010). Anxiety disorders and sensory over-responsivity in children with autism spectrum disorders: is there a causal relationship? Journal of Autism and Developmental Disorders, 40(12), 1495-1504. doi:10.1007/s10803-0101007-x

Green, S. A., Ben-Sasson, A., Soto, T. W., \& Carter, A. S. (2011). Anxiety and Sensory OverResponsivity in Toddlers with Autism Spectrum Disorders: Bidirectional Effects Across Time. Journal of Autism and Developmental Disorders, 42(6), 1112-1119. doi:10.1007/s10803-011-1361-3

Green, S. A., Rudie, J. D., Colich, N. L., Wood, J. J., Shirinyan, D., Hernandez, L., et al. (2013). Over-Reactive Brain Responses to Sensory Stimuli in Youth With Autism Spectrum Disorders RH: fMRI Response to Sensory Stimuli in ASD. Journal of the American Academy of Child and Adolescent Psychiatry, 52(11). doi:10.1016/j.jaac.2013.08.004

Güçlü, B., Tanidir, C., Mukaddes, N. M., \& Unal, F. (2007). Tactile sensitivity of normal and autistic children. Somatosensory \& Motor Research, 24(1-2), 21-33. doi:10.1080/08990220601179418

Haesen, B., Boets, B., \& Wagemans, J. (2011). A review of behavioural and electrophysiological studies on auditory processing and speech perception in autism spectrum disorders. Research in Autism Spectrum Disorders, 5(2), 701-714. doi:10.1016/j.rasd.2010.11.006

Haigh, S. M., Gupta, A., Barb, S. M., Glass, S. A. F., Minshew, N. J., Dinstein, I., et al. (2016). Differential sensory fMRI signatures in autism and schizophrenia: Analysis of amplitude and trial-to-trial variability. Schizophrenia Research. doi:10.1016/j.schres.2016.03.036

Haker, H., Schneebeli, M., \& Stephan, K. E. (2016). Can Bayesian Theories of Autism Spectrum Disorder Help Improve Clinical Practice? Frontiers in Psychiatry, 7. doi:10.3389/fpsyt.2016.00107

Herdman, M., Fox-Rushby, J., \& Badia, X. (1998). A model of equivalence in the cultural adaptation of HRQoL instruments: the universalist approach. Quality of Life Research: An International Journal of Quality of Life Aspects of Treatment, Care and Rehabilitation, 7(4), 323-335.

Horder, J., Wilson, C. E., Mendez, M. A., \& Murphy, D. G. (2014). Autistic traits and abnormal sensory experiences in adults. Journal of Autism and Developmental Disorders, 44(6), 1461-1469. doi:10.1007/s10803-013-2012-7

Kanner, Leo. (1943). Autistic disturbances of affective contact., Nervous Child, 2, 3, 217 250. 
Kern, J. K., Garver, C. R., Grannemann, B. D., Trivedi, M. H., Carmody, T., Andrews, A. A., \& Mehta, J. A. (2007). Response to vestibular sensory events in autism. Research in Autism Spectrum Disorders, 1(1), 67-74. doi:10.1016/j.rasd.2006.07.006

Kern, J. K., Trivedi, M. H., Grannemann, B. D., Garver, C. R., Johnson, D. G., Andrews, A. A., et al. (2007). Sensory correlations in. Autism, 11(2), 123-134. doi:10.1177/1362361307075702

Kirby, A. V., Dickie, V. A., \& Baranek, G. T. (2014). Sensory experiences of children with autism spectrum disorder: In their own words. Autism, 1362361314520756. doi: $10.1177 / 1362361314520756$

Lawson, R. P., Rees, G., \& Friston, K. J. (2014). An aberrant precision account of autism. Frontiers in Human Neuroscience, 8, 302. doi:10.3389/fnhum.2014.00302

Le Couteur, A., Lord, C., \& Rutter, M. (2003). The autism diagnostic interview-revised (ADIR) (Los Angeles: Western Psychological Services.).

Leekam, S. R., Nieto, C., Libby, S. J., Wing, L., \& Gould, J. (2007). Describing the sensory abnormalities of children and adults with autism. Journal of Autism and Developmental Disorders, 37(5), 894-910. doi:10.1007/s10803-006-0218-7

Lord, C., Risi, S., Lambrecht, L., Cook, E. H., Leventhal, B. L., DiLavore, P. C., et al. (2000). The autism diagnostic observation schedule-generic: a standard measure of social and communication deficits associated with the spectrum of autism. Journal of Autism and Developmental Disorders, 30(3), 205-223.

Lundqvist, L.-O. (2015). Hyper-responsiveness to touch mediates social dysfunction in adults with autism spectrum disorders. Research in Autism Spectrum Disorders, 9, 13-20. doi:10.1016/j.rasd.2014.09.012

Lundqvist, L.-O., \& Lindner, H. (2017). Is the Autism-Spectrum Quotient a Valid Measure of Traits Associated with the Autism Spectrum? A Rasch Validation in Adults with and Without Autism Spectrum Disorders. Journal of Autism and Developmental Disorders, 47(7), 2080. doi:10.1007/s10803-017-3128-y

Marco, E. J., Hinkley, L. B. N., Hill, S. S., \& Nagarajan, S. S. (2011). Sensory Processing in Autism: A Review of Neurophysiologic Findings: Pediatric Research, 69(5 Part 2), 48R-54R. doi:10.1203/PDR.0b013e3182130c54

Markram, H., Rinaldi, T., \& Markram, K. (2007). The intense world syndrome--an alternative hypothesis for autism. Frontiers in Neuroscience, 1(1), 77-96. doi:10.3389/neuro.01.1.1.006.2007

Markram, K., \& Markram, H. (2010). The Intense World Theory - A Unifying Theory of the Neurobiology of Autism. Frontiers in Human Neuroscience, 4. doi:10.3389/fnhum.2010.00224

Milne, E., Dickinson, A., \& Smith, R. (2017). Adults with autism spectrum conditions experience increased levels of anomalous perception. PloS One, 12(5), e0177804. doi:10.1371/journal.pone.0177804

Mottron, L, \& Burack, J. (2001). Enhanced perceptual functioning in the development of autism. In The development of autism: Perspectives from theory and research (Burack, Charman, Yirmiya, Zelazo., pp. 131-148). 
Mottron, L., Dawson, M., Soulières, I., Hubert, B., \& Burack, J. (2006). Enhanced perceptual functioning in autism: an update, and eight principles of autistic perception. Journal of autism and developmental disorders, 36(1), 27-43. doi:10.1007/s10803-005-0040-7

Neil, L., Olsson, N. C., \& Pellicano, E. (2016). The Relationship Between Intolerance of Uncertainty, Sensory Sensitivities, and Anxiety in Autistic and Typically Developing Children. Journal of Autism and Developmental Disorders, 46(6), 1962-1973. doi:10.1007/s10803-016-2721-9

Palmer, C. J., Lawson, R. P., \& Hohwy, J. (2017). Bayesian Approaches to Autism: Towards Volatility, Action, and Behavior. Psychological Bulletin. doi:10.1037/bul0000097

Pellicano, E., \& Burr, D. (2012). When the world becomes "too real": a Bayesian explanation of autistic perception. Trends in cognitive sciences, 16(10), 504-510. doi:10.1016/j.tics.2012.08.009

Puts, N. A. J., Wodka, E. L., Tommerdahl, M., Mostofsky, S. H., \& Edden, R. A. E. (2014). Impaired tactile processing in children with autism spectrum disorder. Journal of Neurophysiology, 111(9), 1803-1811. doi:10.1152/jn.00890.2013

Revelle, W. (1978). ICLUST: A cluster analytic approach to exploratory and confirmatory scale construction. Behavior Research Methods \& Instrumentation, 10(5), 739-742. doi:10.3758/BF03205389

Revelle, W. (1979). Hierarchical Cluster Analysis And The Internal Structure Of Tests. Multivariate Behavioral Research, 14(1), 57-74. doi:10.1207/s15327906mbr1401_4

Riquelme, I., Hatem, S. M., \& Montoya, P. (2016). Abnormal Pressure Pain, Touch Sensitivity, Proprioception, and Manual Dexterity in Children with Autism Spectrum Disorders. Neural Plasticity, 2016, 1723401. doi:10.1155/2016/1723401

Robertson, A. E., \& David R Simmons, R. (2015). The sensory experiences of adults with autism spectrum disorder: A qualitative analysis. Perception, 44(5), 569-586.

Robertson, A. E., \& Simmons, D. R. (2013). The Relationship between Sensory Sensitivity and Autistic Traits in the General Population. Journal of Autism and Developmental Disorders, 43(4), 775-784. doi:10.1007/s10803-012-1608-7

Robic, S., Sonié, S., Fonlupt, P., Henaff, M.-A., Touil, N., Coricelli, G., et al. (2014). Decision-Making in a Changing World: A Study in Autism Spectrum Disorders. Journal of Autism and Developmental Disorders. doi:10.1007/s10803-014-2311-7

Simmons, D., \& Milne, E. (2015). Response to Davis and Plaisted-Grant: Low or high endogenous neural noise in autism spectrum disorder? Autism: The International Journal of Research and Practice, 19(3), 363-364. doi:10.1177/1362361314557683

Simmons, D. R., McKay, L., McAleer, P., Toal, E., Robertson, A., \& Pollick, F. E. (2007). Neural noise and autism spectrum disorders. Perception, 36, 119-120.

Simmons, D. R., Robertson, A. E., McKay, L. S., Toal, E., McAleer, P., \& Pollick, F. E. (2009). Vision in autism spectrum disorders. Vision research, 49(22), 2705-2739. doi:10.1016/j.visres.2009.08.005

Sinclair, D., Oranje, B., Razak, K. A., Siegel, S. J., \& Schmid, S. (2016). Sensory processing in autism spectrum disorders and Fragile $\mathrm{X}$ syndrome-From the clinic to animal models. Neuroscience and Biobehavioral Reviews. doi:10.1016/j.neubiorev.2016.05.029 
Smith, R. S., \& Sharp, J. (2013). Fascination and isolation: a grounded theory exploration of unusual sensory experiences in adults with Asperger syndrome. Journal of Autism and Developmental Disorders, 43(4), 891-910. doi:10.1007/s10803-012-1633-6

Sonié, S., Kassai, B., Pirat, E., Bain, P., Robinson, J., Gomot, M., et al. (2013). The French version of the autism-spectrum quotient in adolescents: a cross-cultural validation study. Journal of Autism and Developmental Disorders, 43(5), 1178-1183. doi:10.1007/s10803-012-1663-0

Sonié, S., Kassai, B., Pirat, E., Masson, S., Bain, P., Robinson, J., et al. (2011). [French version of screening questionnaire for high-functioning autism or Asperger syndrome in adolescent: Autism Spectrum Quotient, Empathy Quotient and Systemizing Quotient. Protocol and questionnaire translation]. Presse Médicale (Paris, France: 1983), 40(4 Pt 1), e181-188. doi:10.1016/j.lpm.2010.07.016

Steiger, J. H. (1980). Testing Pattern Hypotheses On Correlation Matrices: Alternative Statistics And Some Empirical Results. Multivariate Behavioral Research, 15(3), 335-352. doi:10.1207/s15327906mbr1503_7

Takayama, Y., Hashimoto, R., Tani, M., Kanai, C., Yamada, T., Watanabe, H., et al. (2014). Standardization of the Japanese version of the Glasgow Sensory Questionnaire (GSQ). Research in Autism Spectrum Disorders, 8(4), 347-353. doi:10.1016/j.rasd.2013.12.017

Tavassoli, T., \& Baron-Cohen, S. (2012). Taste identification in adults with autism spectrum conditions. Journal of Autism and Developmental Disorders, 42(7), 1419-1424. doi:10.1007/s10803-011-1377-8

Tavassoli, T., Hoekstra, R. A., \& Baron-Cohen, S. (2014). The Sensory Perception Quotient (SPQ): development and validation of a new sensory questionnaire for adults with and without autism. Molecular Autism, 5, 29. doi:10.1186/2040-2392-5-29

Tavassoli, T., Miller, L. J., Schoen, S. A., Nielsen, D. M., \& Baron-Cohen, S. (2014). Sensory over-responsivity in adults with autism spectrum conditions. Autism: The International Journal of Research and Practice, 18(4), 428-432. doi:10.1177/1362361313477246

Van de Cruys, S., de-Wit, L., Evers, K., Boets, B., \& Wagemans, J. (2013). Weak priors versus overfitting of predictions in autism: Reply to Pellicano and Burr (TICS, 2012). I-Perception, 4(2), 95-97. doi:10.1068/i0580ic

Van de Cruys, S., Evers, K., Van der Hallen, R., Van Eylen, L., Boets, B., de-Wit, L., \& Wagemans. (2014). Precise minds in uncertain worlds: Predictive coding in autism. https://irias.kuleuven.be/handle/123456789/455945. Accessed 17 July 2014

Van de Cruys, S., Van der Hallen, R., \& Wagemans, J. (2016). Disentangling signal and noise in autism spectrum disorder. Brain and Cognition. doi:10.1016/j.bandc.2016.08.004

Velicer, W. F. (1976). Determining the number of components from the matrix of partial correlations. Psychometrika, 41(3), 321-327. doi:10.1007/BF02293557

Ward, J., Hoadley, C., Hughes, J. E. A., Smith, P., Allison, C., Baron-Cohen, S., \& Simner, J. (2017). Atypical sensory sensitivity as a shared feature between synaesthesia and autism. Scientific Reports, 7, 41155. doi:10.1038/srep41155 
Wicker, B., Monfardini, E., \& Royet, J.-P. (2016). Olfactory processing in adults with autism spectrum disorders. Molecular Autism, 7, 4. doi:10.1186/s13229-016-0070-3

Zachor, D. A., \& Ben-Itzchak, E. (2013). The Relationship Between Clinical Presentation and Unusual Sensory Interests in Autism Spectrum Disorders: A Preliminary Investigation. Journal of Autism and Developmental Disorders. doi:10.1007/s10803013-1867-y 


\section{Tables}

Table 1: Demographic data and descriptive statistics for the AQ and GSQ

\begin{tabular}{|c|c|c|c|c|c|}
\hline \multicolumn{6}{|c|}{ Demographic data } \\
\hline & & All subjects & Low AQ group & High AQ group & $\begin{array}{c}\text { Low vs. high } \\
\text { AQ group }\end{array}$ \\
\hline \multicolumn{2}{|c|}{ Number } & 245 & 143 & 102 & - \\
\hline \multicolumn{2}{|c|}{ Females/Males } & $114 / 131$ & $58 / 85$ & $56 / 46$ & $n s$ \\
\hline \multicolumn{2}{|l|}{ Age } & $32.1( \pm 10.8)$ & $30.7( \pm 11.1)$ & $34.2( \pm 10.2)$ & $*$ \\
\hline \multicolumn{2}{|c|}{ Educational level } & $13.7( \pm 1.1)$ & $14.1( \pm 0.9)$ & $13.3( \pm 1.2)$ & $* *$ \\
\hline \multicolumn{2}{|c|}{$A Q$} & $23.0( \pm 13.8)$ & $12.4( \pm 5.1)$ & $38.0( \pm 6.0)$ & $* * *$ \\
\hline \multirow[t]{3}{*}{$G S Q$} & Total & $58.7( \pm 28.4)$ & $41.6( \pm 14.7)$ & $82.8( \pm 25.4)$ & $* * *$ \\
\hline & Hypersensitivity & $32.3( \pm 16.9)$ & $21.9( \pm 8.4)$ & $46.9( \pm 14.9)$ & $* * *$ \\
\hline & Hyposensitivity & $26.4( \pm 12.9)$ & $19.6( \pm 8.0)$ & $35.9( \pm 12.4)$ & $* * *$ \\
\hline
\end{tabular}

Numbers corresponds to the mean ( \pm standard deviation), in all participants, in the low AQ and high AQ groups (using a cut-off of 26). Education level corresponds to the number of year of formal education (from the first year of elementary school).

The right column corresponds to results from Student t-test comparing the two groups.

${ }^{*} p<.05,{ }^{* *} p<10^{-3},{ }^{* * *} p<10^{-6}$. ns: not significant $(p>.05)$.

AQ: Autism-Spectrum Quotient, GSQ: Glasgow Sensory Questionnaire. 
Table 2: Correlations between GSQ and AQ subscales

\begin{tabular}{|c|c|c|c|c|}
\hline \multicolumn{5}{|c|}{ Correlations between GSQ and $A Q$ subscales } \\
\hline & $\begin{array}{c}\text { All subjects } \\
r\end{array}$ & $\begin{array}{c}\text { Low AQ } \\
r\end{array}$ & $\begin{array}{c}\text { High AQ } \\
r\end{array}$ & $\begin{array}{c}\text { Low vs. high } \\
\text { AQ group }\end{array}$ \\
\hline \multicolumn{5}{|c|}{ Correlations with the total Glasgow Sensory Questionnaire score } \\
\hline$A Q$ Social skills & $.76^{* * *}$ & $.28^{* *}$ & $.47^{* *}$ & $n s$ \\
\hline$A Q$ Attention switching & $.70^{* * *}$ & $.21^{*}$ & $.38^{* *}$ & $n s$ \\
\hline$A Q$ Attention to detail & $.66^{* * *}$ & $.38^{* *}$ & $.47^{* * *}$ & $n s$ \\
\hline$A Q$ Communication & $.77^{* * * *}$ & $.28^{* *}$ & $.52^{* * *}$ & $<.05$ \\
\hline$A Q$ Imagination & $.61^{* * *}$ & .13 & .21 & ns \\
\hline \multicolumn{5}{|c|}{ Correlations with the Autism-Spectrum quotient } \\
\hline$G S Q$ Hypersensitivity & $.81^{* * *}$ & $.37^{* *}$ & $.61^{* * *}$ & $<.01$ \\
\hline GSQ Hyposensitivity & $.72^{* * *}$ & $.44^{* * *}$ & $.54^{* * *}$ & $n s$ \\
\hline$G S Q$ Visual & $.71^{* * *}$ & $.39^{* *}$ & $.56^{* * *}$ & $n s$ \\
\hline GSQ Auditory & $.80^{* * *}$ & $.40^{* * *}$ & $.51^{* * *}$ & $n s$ \\
\hline$G S Q$ Gustatory & $.58^{* * *}$ & $.28^{* *}$ & $.45^{* *}$ & $n s$ \\
\hline$G S Q$ Olfactory & $.64^{* * *}$ & .12 & $.45^{* *}$ & $<.01$ \\
\hline$G S Q$ Tactile & $.71^{* * *}$ & $.30^{* *}$ & $.54^{* * *}$ & $<.05$ \\
\hline$G S Q$ Vestibular & $.71^{* * *}$ & $.41^{* * *}$ & $.47^{* * *}$ & $n s$ \\
\hline GSQ Proprioceptive & $.76^{* * *}$ & $.39^{* *}$ & $.57^{* * * *}$ & $n s$ \\
\hline
\end{tabular}

Correlations between GSQ and AQ subscores in all subjects, in the low AQ and in the high AQ groups. Pearson correlation tests: ${ }^{*} p<.01,{ }^{* *} p<10^{-3},{ }^{* * *} p<10^{-6}$ (non-significant for $p>$ .01 for the five AQ subscales, $p>.025$ for the two hypersensitivity and hyposensitivity scales, $p>.007$ for the seven sensory GSQ subscales, after Bonferroni correction).

Correlation coefficients were compared between groups using a Fisher r-to-z transformation and results are shown in the right column $n s=$ non-significant. 
Table 3: Correlations between the GSQ subscores of hyper- and hypo-sensitivity

\begin{tabular}{lcccc}
\hline \multicolumn{5}{c}{ Correlations between } \\
& All subjects & Low AQ & High AQ & Low vs. high \\
GSQ subscales & $\boldsymbol{r}$ & $\boldsymbol{r}$ & $\boldsymbol{r}$ & AQ group \\
\hline Total & $0.82^{* * *}$ & $0.60^{* * *}$ & $0.73^{* * *}$ & $n s(.07)$ \\
Visual & $0.60^{* * *}$ & $0.31^{* *}$ & $0.54^{* * *}$ & .03 \\
Auditory & $0.57^{* * *}$ & $0.23^{* * *}$ & $0.30^{*}$ & $n s$ \\
Gustatory & $0.36^{* * *}$ & 0.23 & $0.45^{* *}$ & $n s(.06)$ \\
Olfactory & $0.25^{* *}$ & 0.09 & 0.21 & $n s$ \\
Tactile & $0.57^{* * *}$ & 0.15 & $0.33^{* *}$ & $n s$ \\
Vestibular & $0.54^{* * *}$ & 0.18 & $0.46^{* *}$ & $<.05$ \\
Proprioceptive & $0.63^{* * *}$ & $0.38^{* *}$ & $0.45^{* *}$ & $n s$ \\
\hline Correlations & & &
\end{tabular}

Correlations between the hypersensitivity and hyposensitivity subscores for all subjects, for the low AQ and the high AQ groups. Pearson correlation tests: ${ }^{*} p<.007,{ }^{* *} p<10^{-3},{ }^{* * *} p<$ $10^{-6}$ (non-significant for $p>.007$, after Bonferroni correction).

Correlation coefficients were compared between groups using a Fisher r-to-z transformation. $n s=$ non-significant. 


\section{Figures}
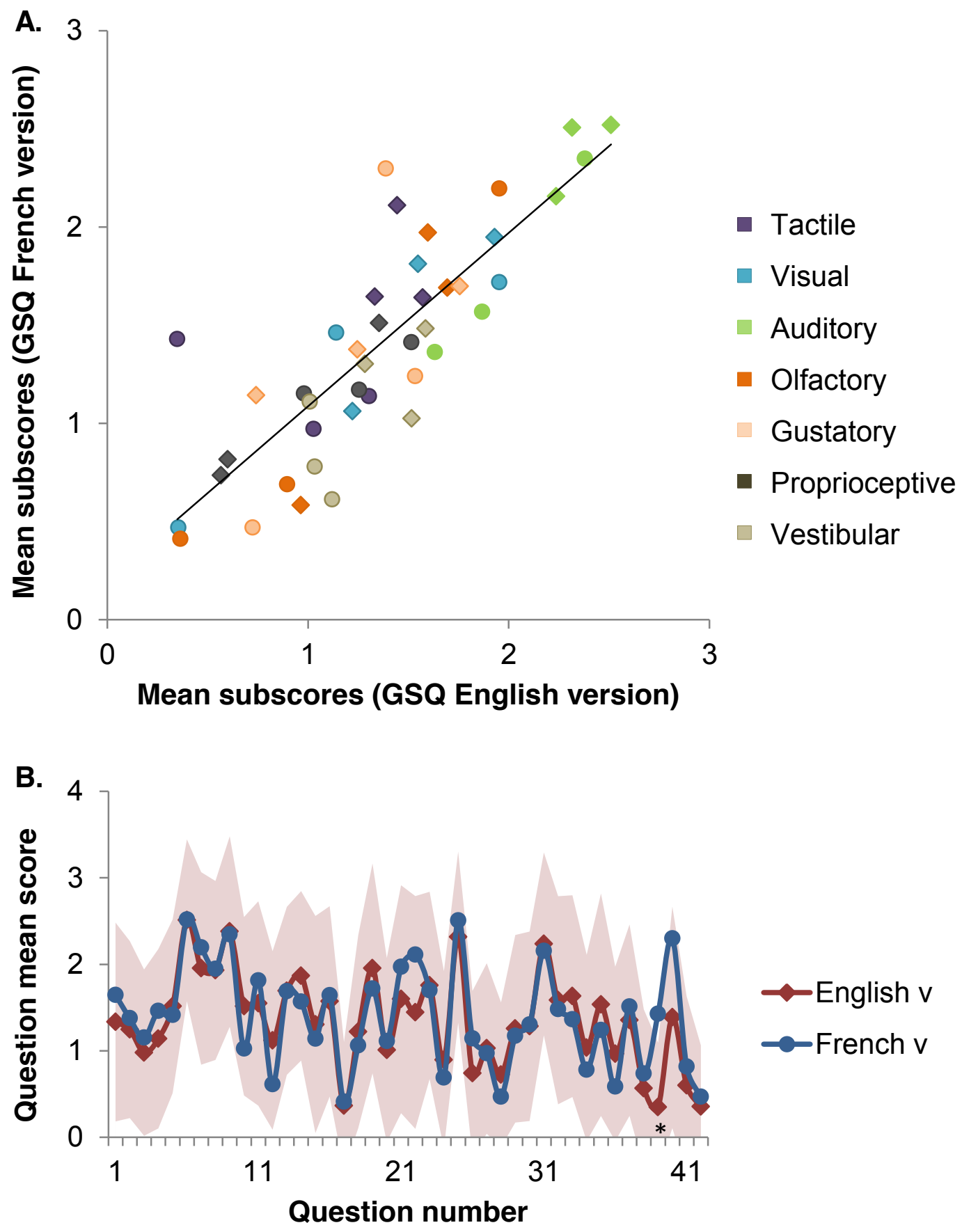

Figure 1: Correlation between the English and the French versions of the GSQ

A. Correlation between scores of the English and French versions of the GSQ. Each sensory modality is represented by a color. Diamonds correspond to questions investigating 
hypersensitivity, while circles correspond to questions investigating hyposensitivity. Pearson correlation: $r=.83, p<10^{-6}$.

B. Scores for each question of the English version of the GSQ (red) and the French version of the GSQ (blue). The red shade area corresponds to the standard deviation for each item in the English version.

* Significant difference between the two versions for question $n^{\circ} 39$. 

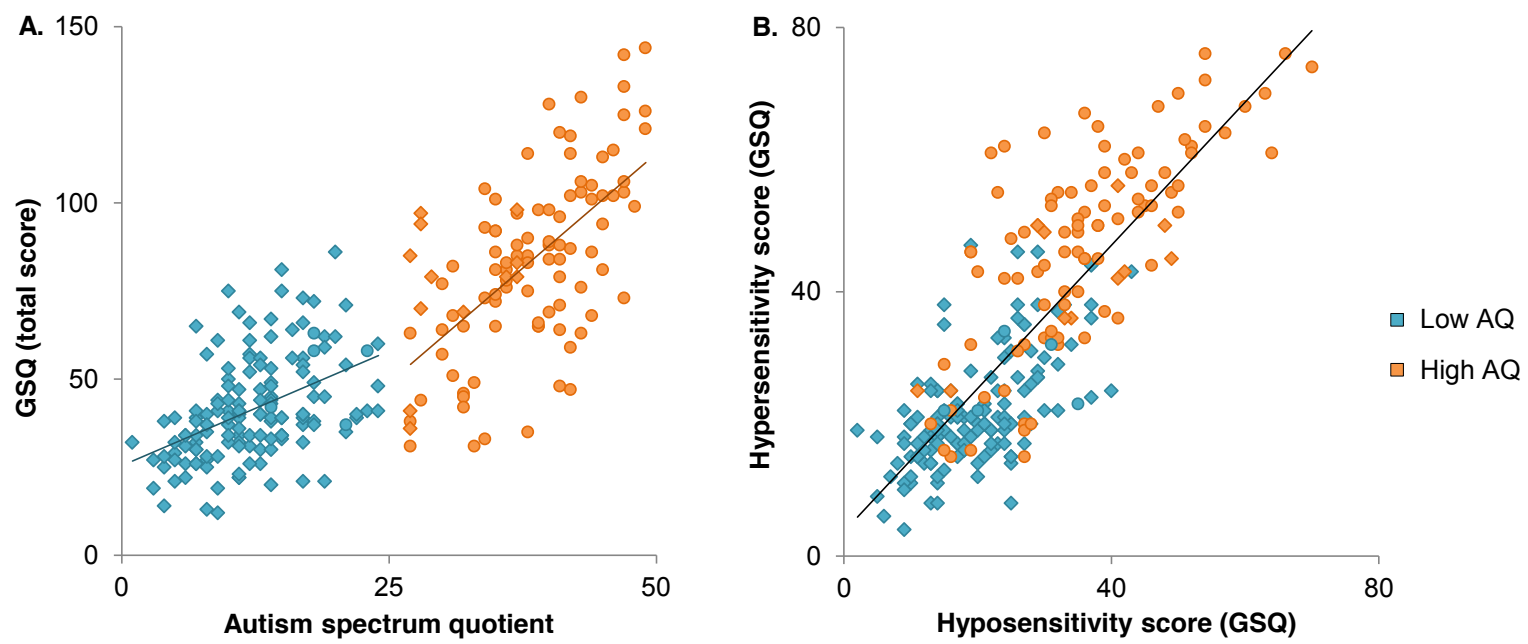

Figure 2: Correlations with the Glasgow Sensory Questionnaire

A. Positive linear correlation between the GSQ total score and the AQ in participants with low AQ (blue) or high AQ (orange).

Linear regression in the low AQ group: $r=.45, p<10^{-6}, y=1.3 x+25.5$.

Linear regression in the high AQ group: $r=.62, p<10^{-6}, y=2.6 x-16.0$.

B. Positive correlation between the two main subscales of the GSQ: the hypersensitivity score and the hyposensitivity score in participants with low AQ (blue) or high AQ (orange). Linear regression: $r=.82, p<10^{-6}$.

Circles correspond to participants who reported having a formal diagnosis of ASD, while diamonds correspond to participants who reported having no formal diagnosis of ASD. Note that these diagnoses were not checked, and only depend on the information they volunteered. 

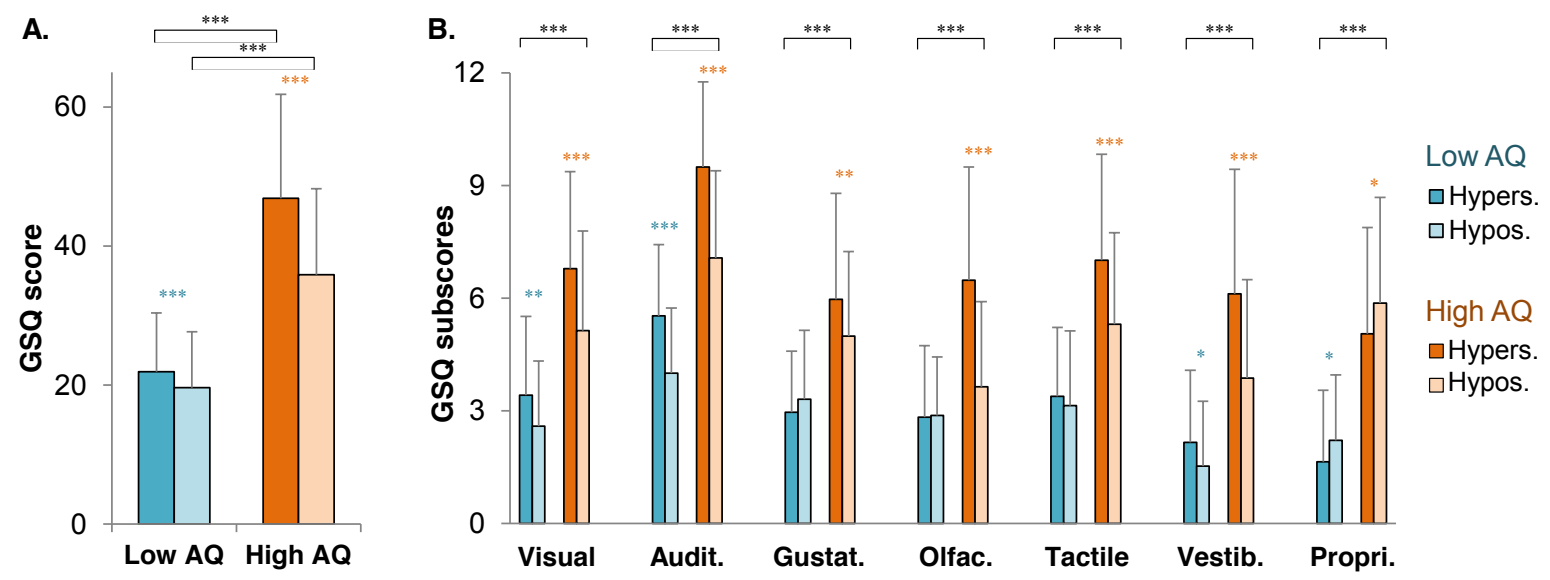

Figure 3: Glasgow sensory questionnaire scores in the low $A Q$ and high $A Q$ groups

A. GSQ scores of hypersensitivity and hyposensitivity in the low AQ (blue) and high AQ (orange) groups.

B. Subscales of the GSQ in the low AQ (blue) and high AQ (orange) groups, for the visual, auditory, gustatory, olfactory, tactile, vestibular and proprioceptive modalities. Black stars indicate significant difference between groups for the total GSQ subscore in each modality.

Clear blue and orange indicate the GSQ scores of hyposensitivity, while dark blue and orange indicate the GSQ scores of hypersensitivity. Blue and orange stars correspond to the significant differences between the hypersensitivity and hyposensitivity subscores within the low AQ group and high AQ group, respectively.

${ }^{*} p<.007,{ }^{* *} p<10^{-3},{ }^{* * *} p<10^{-6}$ (Bonferroni correction for multiple comparisons involving the seven subscales of the GSQ gives a threshold of significance for $p<.007$ ). Error bars correspond to standard deviations. 


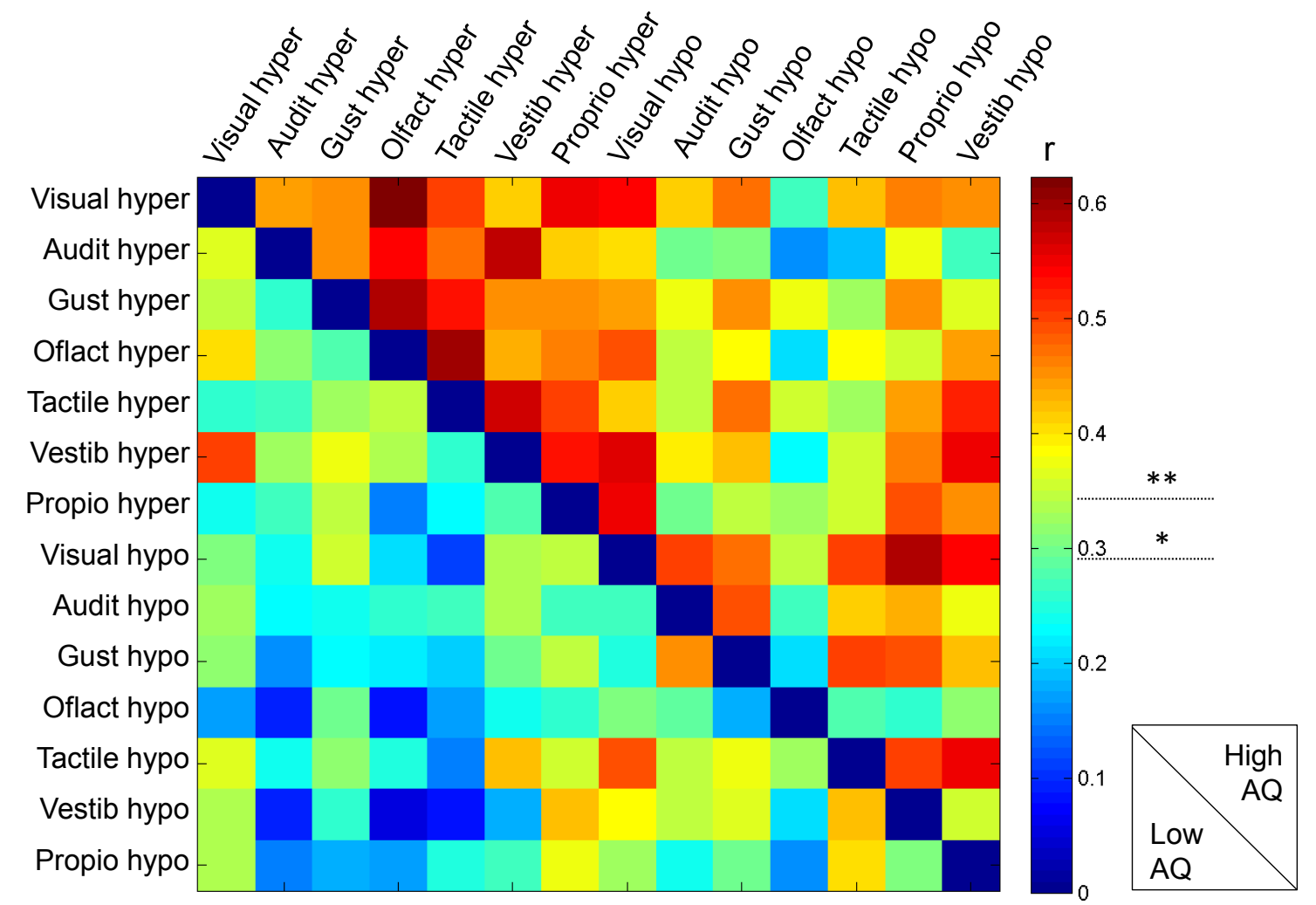

Figure 4: Correlations between the subscores of the GSQ in the low $A Q$ and high $A Q$ groups

The matrix indicates the Pearson correlation coefficients between the subscores of the GSQ in the low AQ group (bottom diagonal) and in the high AQ group (top diagonal). P-values associated with the correlation coefficients are shown with the stars on the colorbar. : ${ }^{*} p<$ $5.10^{-4}$ (adjusted with Bonferroni correction for 91 comparisons), ${ }^{* *} p<5.10^{-5}$.

Audit: Auditory, Gust: Gustatory, Olfact: Olfactory, Vestib: Vestibular, Proprio: Proprioceptive, hyper: hypersensitivity, hypo: hyposensitivity. 


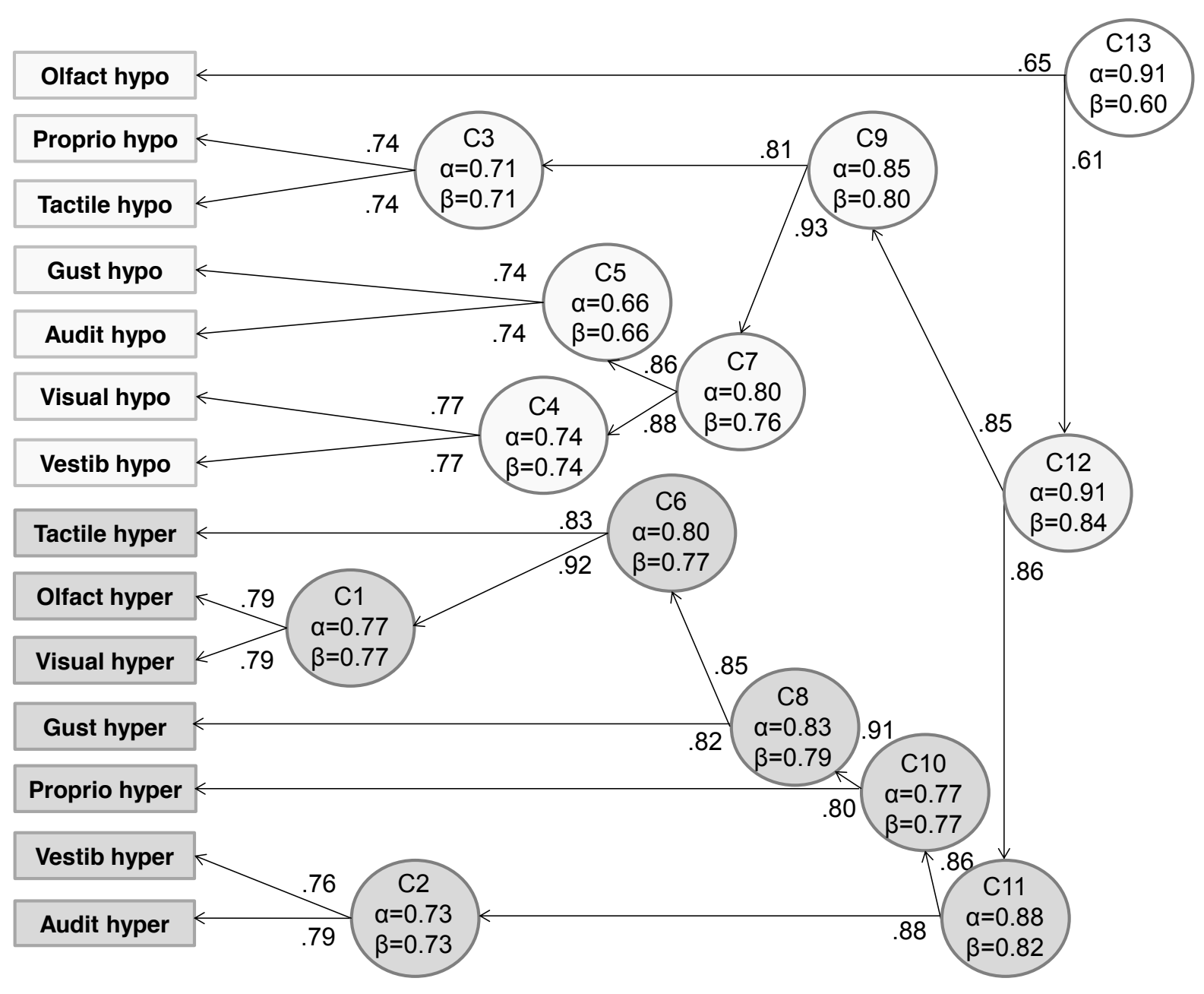

\section{Figure 5: Hierarchical cluster analysis of the 14 GSQ subscales in the high AQ group}

This analysis revealed the cluster $\mathrm{C} 11$ associated with the hypersensitivity subscores, the cluster C9 associated with the hyposensitivity subscores. The main cluster C12 gathers the hypersensitivity and hyposensitivity clusters. The addition of the olfactory hyposensitivity item in cluster $\mathrm{C} 13$ led to a drop in homogeneity $(\beta=.6)$ suggesting a poor fit of this item. The equivalent pattern for the low AQ group is shown in Appendix 2 (Figure SI-A).

Audit: Auditory, Gust: Gustatory, Olfact: Olfactory, Vestib: Vestibular, Proprio: Proprioceptive, hyper: hypersensitivity, hypo: hyposensitivity. 